\title{
New Trends in the Development of Treatments of Viral Myocarditis
}

\author{
Decheng Yang, Huifang Mary Zhang, Xin Ye, \\ Lixin Zhang and Huanqin Dai \\ Additional information is available at the end of the chapter \\ http://dx.doi.org/10.5772/54103
}

\section{Introduction}

Viral myocarditis is caused by a variety of viruses of more than 10 genera, such as coxsackievirus, adenovirus, parvovirus, hepatitis c virus, herpes virus, influenza virus, HIV, etc. [1]. However, the most frequently reported and extensively studied one is coxsackievirus B3 (CVB3), which causes $\sim 30 \%$ of all viral myocarditis cases [2]. Thus, in this chapter the review will mainly focus on CVB3-induced myocarditis. This virus can infect multiple organs of human such as heart, pancreas, brain, liver, lung, spleen, etc. and cause myocarditis, pancreatitis, meningitis, hepatitis, etc. However, the most fatal disease is myocarditis, particular in children and young people [3]. Viral myocarditis is characterized by inflammatory infiltration of immune cells in the heart muscle after viral infection. This viral infection can cause direct damage of cardiomyocytes as well as immune-mediated destructions of the myocardium, leading to cardiac dysfunction. In addition, viral myocarditis often progresses into dilated cardiomyopathy (DCM), an end-stage heart dysfunction. Patients with DCM usually require heart transplantation [4]. There is no other treatment option at the present. Viral myocarditis is one of the major life-threatening diseases in children. It is the cause of $\sim 20 \%$ of sudden unexpected death in young people [5]. To date, there is no specific treatment for this viral infection.

CVB3 is a positive single-stranded, non-enveloped RNA virus of the enterovirus genus of the Picornaviridae family. Its genome is $\sim 7.4 \mathrm{~kb}$ long, containing a single long open reading frame (encoding 11 proteins) flanked by the $5^{\prime}$ and $3^{\prime}$ untranslated regions (UTRs). The 5' UTR is 741 nucleotides (nt) long and harbors a number of cis-acting translational elements, such as the internal ribosomal entry site (IRES) and the cloverleaf sequence [6-9], which are crucial structures for viral translation and transcription. The 3' UTR is a 99-nt long segment attached with a poly-A tail. The 3' UTR folds to form kissing-loop tertiary structures, which 
are believed to play a role in facilitating viral transcription of the negative strand of CVB3 replication intermediate $[10,11]$. The viral genomic RNA can directly serve as a mRNA template for translation of a single long polyprotein, which is processed by viral proteases to produce eleven individual proteins, among which four are structural proteins, VP1-VP4, and seven are non-structural proteins including proteases $2 \mathrm{~A}$ and $3 \mathrm{C}$, as well as a RNA-dependent RNA polymerase 3D. These three enzymatic proteins play important roles in viral life cycle and pathogenesis.

CVB3 infects cardiomyocytes by endocytosis through viral receptor CAR (coxsackie and adenovirus receptor) co-localized with tight junction proteins (e.g., occludin) [12]. It is also known that CAR-binding site (anti-receptor) on CVB3 particle lies in the canyon on the capsid surface. Upon attachment of CVB3 particles to CAR, the receptor changes conformation to form the viral A-particle, a product of the interactions between CVB3 and CAR, which then allows for the release of viral RNA into host cells and begins viral translation and transcription. The observation that soluble CAR protein can function as a virus trap leading to inactive A-particles has suggested a strategy for CVB3 therapy [13-15]. Depending on the different combination of viral strains and mouse models in the study of CVB3 infection, a CVB3 co-receptor called decay accelerating factor (DAF, CD55) is sometimes also necessary for CVB3 entry into the host cells $[16,17]$. Thus, genes encoding CAR and DAF are important candidates for study of viral tropism and rational targets for antiviral drug design.

In recent years, extensive researches have been conducted for drug development. Although effective treatments are still not clinically available for this viral disease, some research strategies are very promising and have made exciting progresses. This chapter will first briefly summarize the current treatments used clinically for viral myocarditis even though they are not very specific and effective. Then we will focus on recent advances in new drug development, which include nucleic acid (NA)-based strategies, natural compounds, cell-based therapy, etc. We will also briefly discuss the limitations and challenges faced by the development of such treatments.

\section{Current treatments}

To date, there is no clinically proven specific treatment for viral myocarditis and DCM. Patients with DCM eventually need heart transplantation as the final option [18]. Managements for viral myocarditis are usually supportive therapies, such as improvements in hemodynamics with drugs used to treat other kinds of heart diseases, and application of non-specific antiviral agents to decrease viral load. The former include administration of angiotensin-converting enzyme inhibitors or angiotensin receptor blockade, beta-adrenergic blockade, diuretics, etc. [18-20]. The latter include application of type I interferon or nucleotide analogs such as ribavirin, which was reviewed elsewhere [3, 18, 19, 21, 22]. If it is caused by an autoimmune disorder, myocarditis would be appropriately treated by immunosuppression $[18,20]$. However, the effectiveness of treatment with immunosuppressive therapies has not reached a consensus amongst different studies. This can probably be at- 
tributed to the difficulty of confirmation and diagnosis of the etiology and pathogenesis of myocarditis. Thus, it is very important to distinguish between infectious and autoimmune disease, since the same methods of treatment will not be optimal for both forms of heart muscle diseases. The diagnostic gold standard is endomyocardial biopsies with the histological Dallas criteria, in association with new immunohistochemical and viral PCR analyses of cardiac tissues [23]. In case of confirmed autoimmune-related disease and lack of detectable viral infection, an immunosuppressive treatment combining corticoids and azathioprine may be beneficial [24]. However, if the disease is primarily caused by viral infections, more specific antiviral agents would be the ideal drugs of choice.

In recent years, the search for such antiviral drugs has become a new trend in drug development for treatment of viral myocarditis. One of the strategies for developing such antivirals is the screening of chemical compounds, such as pleconaril, capable of interacting with picornavirus (particularly human rhinovirus) anti-receptor to block viral entry into the host cells [25-27]. Pleconaril functions in a mechanism similar to that of WIN compounds, by interacting with the hydrophobic amino acid residues located within the canyon floor of the anti-receptor of host cell. Thus, it results in the blockage of the attachment of viral particles to the host cell surface and reduces viral load in the heart [28]. Furthermore, the binding of WIN compounds also results in increased protein rigidity and stabilizes the entire viral capsid against enzymatic degradation, so that viral uncoating and release of viral RNA into the cytoplasm is inhibited [29, 30]. Pleconaril was initially developed for treatment of human common cold caused by human rhinovirus, a close relative of CVB3. It also shows effectiveness in inhibiting CVB3 infection [31]. To avoid mutation escape induced by pleconaril, new pleconaril derivatives have been synthesized and successfully tested against pleconaril-resistant mutants [32]. However, due to its high toxicity, pleconaril has not passed the approval by FDA of USA and is only used in a compassionate manner.

\section{New strategies in drug development}

\subsection{Nucleic acid (NA)-based antivirals against CVB3 infection}

\subsubsection{Anti-CVB3 antisense oligonucleotides (ASONs)}

ASONs are designed to bind to a complementary sequence in the target mRNA to form RNADNA heteroduplexes. These double-stranded hybrid sequences are recognized by RNase $\mathrm{H}$, which digests the RNA strand in the duplex. Due to major problems, including instability, nonspecific delivery, and unwanted side effects of the ASONs, the structure of this molecule has been modified extensively at different components (i.e., bases, sugar, or phosphate backbone), and has entered its third generation. The first generation of chemical modification was designed to enhance nuclease resistance of ASON in serum [33]. The representative of such is the phosphorothioate (PS) oligonucleotide (ON), in which one of the non-bridging oxygen atoms in the phosphodiester bond is replaced by sulfur, intended to prevent cleavage by nucleases. Early antiviral PS-modified ASONs exhibited the antisense properties of phosphodiester 
ASONs, such as the ability to induce RNase $\mathrm{H}$ activation, while showing enhanced stability [34]. Another strategy to increase the stability of ASONs is the addition of alkyl groups at the 2 position of the ribose. 2-O-methyl (OMe) and 2-O-methoxy-ethyl (MOE) substitutions sterically shield the backbone from nuclease access, and also increase affinity to the target [35]. These modified ASONs function mainly by blocking translation via steric hindrance of elongating ribosome but not by RNAse H-mediated cleavage. In order to retain the advantage of the RNAse $\mathrm{H}$ mechanism, chimeric oligos containing both 2 unmodified and 2-modified DNAs, called gapmers, were conceived. The 2-O-alkyl modified ASONs and mixed backbone gapmer ASONs represent a second generation of ASON. The third generation ASONs are phosphorodiamidate morpholino oligonucleotides (PMOs). PMOs have a structure in which the ribose is replaced by a morpholine moiety and phosphorodiamidate (O-PONH2-O) linkers are used instead of phosphodiester bonds. Thus, PMOs are resistant to digestion by nucleases and are electrically neutral. PMO-RNA hybrids do not activate RNase H. Therefore, the mechanism by which PMOs inhibit protein synthesis is via binding the critical mRNA elements, such as the mRNA 5'UTR or the start codon region, to prevent ribosomes from binding or scanning.

CVB3, one of the most frequently used model systems for study of viral replication and pathogenesis, is also widely employed for evaluation of NA-based antiviral agents. The early investigations mainly focused on the application of the second and third generations of ASONs. McManus and coworkers are one of the pioneer groups to study the potential possibility to inhibit CVB3 replication using ASONs. Their earliest work using regular ASONs to target the different sites of 5' UTR of CVB3 genome successfully mapped the IRES by in vitro translation inhibition assay [9]. That study provided useful information for the design of ASON for inhibiting CVB3 replication in vitro and in mouse models. Later, they used PSASONs targeting the $5^{\prime}$ and $3^{\prime}$ UTRs as well as the start codon region, and found that the oligomers targeting the $5^{\prime}$ and $3^{\prime}$ proximate ends of the CVB3 genome are the most effective candidates to inhibit viral replication in HeLa cells. Each of these two ASONs resulted in $\sim 80 \%$ reduction of viral particle production, which is followed by the candidates targeting the IRES and the initiation codon region [36]. The importance of these sites for ASON binding was further confirmed by in vivo evaluation using a murine myocarditis model, although the antiviral efficiency is not as high as that obtained from in vitro evaluation [37].

To improve the stability of the oligomers, our group designed eight PMOs targeting both the sense and antisense strands of the CVB3 replication intermediate. To increase the efficiency of drug internalization, the PMOs were conjugated to a cell-penetrating arginine-rich peptide. These modified ASONs were evaluated in HeLa cells and HL-1 cardiomyocytes in culture and in a murine myocarditis model [38]. One of the oligomers, designed to target a sequence in the $3^{\prime}$ portion of the CVB3 IRES, was found to be especially potent against CVB3. Treatment of cells with this oligomer prior to CVB3 infection produced an approximately 3-log10 decrease in viral titer and largely protected cells from virus-induced cytopathic effect. A similar antiviral effect was observed when this oligomer treatment began shortly after the virus infection period. A/J mice receiving intravenous administration of this oligomer once prior to and once after CVB3 infection showed an $\sim 2-\log 10$-decreased viral titer in the myocardium at 7 days post infection and a significantly decreased level of cardiac tissue damage, compared to the controls [38]. 
In addition to the many ASON reports, another strategy using CpG containing oligodeoxynucleotide to activate antiviral immunity has been reported [39]. The mechanism is that the C-type of CpG oligomer can induce anti-CVB3 activity in human peripheral blood mononuclear cells through the induction of synthesis of natural mixed interferons.

\subsubsection{Antiviral ribozymes}

Ribozymes are catalytically active small RNA ( 30-100 nts) molecules that act as enzymes to specifically cleave single strand RNA without the need of proteins. A major therapeutic advantage of ribozymes is the ability to make them trans-acting and to confer specificity to virtually cleave any target sequence [40]. This can be achieved by fusing the ribozyme core sequence at the $5^{\prime}$ and $3^{\prime}$ ends with the sequences that are complementary to the target sequence.

Ribozyme as an antiviral agent has been tested for many viral infections; however, report on anti-CVB3 has not been documented. Here, we will take HCV as an example to briefly discuss the potential application of ribozyme for the treatment of $\mathrm{HCV}$ infection, as many recent reports found that $\mathrm{HCV}$ is a new causal agent of myocarditis [41, 42]. To investigate the potential application of synthetic, stabilized ribozymes for the treatment of chronic HCV infection, Macejak et al. designed and synthesized hammerhead ribozymes targeting 15 conserved sites in the 5' UTR of HCV RNA including the IRES [43]. It was shown that the inhibitory activity of ribozyme targeting site at nt 195 of HCV RNA exhibited a sequence-specific dose response, required an active catalytic ribozyme core, and was dependent on the presence of the HCV $5^{\prime}$ UTR. In an investigation of new genetic approaches on the management of this infection, six hammerhead ribozymes directed against a conserved region of the plus strand and minus strand of the HCV genome were isolated from a ribozyme library that was expressed using recombinant adenovirus vectors [44]. Treatment with synthetic stabilized anti-HCV ribozymes and vector-expressed HCV ribozymes has the potential to aid in treatment of patients who are infected with $\mathrm{HCV}$ by reducing the viral burden through specific targeting and cleavage of the viral genome. Gonzalez-Carmona and colleagues used RNA transcripts from a construct encoding a HCV-5'-NCR-luciferase fusion protein to test four chemically modified HCV specific ribozymes in a cell-free system and in HepG2 or CCL13 cell lines. They found that ribozyme (Rz1293) showed an inhibitory activity of viral translation of more than $70 \%$, thus verifying that the GCA 348 cleavage site in the HCV loop IV is an accessible target site in cell culture and may be suitable for the development of novel optimized hammerhead structures [45].

\subsubsection{Anti-CVB3 siRNAs}

Accumulated evidence suggests that RNA interference (RNAi) plays an important role in the antiviral defense mechanism in mammalian cells [46-49]. These findings fueled the interests of researchers to use RNAi for antiviral drug development $[49,50]$.

The specificity of RNA silencing is mediated by small RNAs called short interfering RNAs (siRNA) and microRNA (miRNA). Both types of RNAs are generated by processing of ribonucleases in the Dicer family, a group of class III endoribonucleases, which cleaves double stranded non-coding RNA into fragments with a length of 21-25 nts. For siRNA, the long dsRNA or 
transgene-expressed short hairpin RNA (shRNA) are cleaved by Dicer. These RNAs are assembled into a multi-component complex, known as the RNA-induced silencing complex (RISC), which incorporates a single strand (antisense strand) of the siRNA serving as a guide sequence to silence the target gene [51,52] (Figure. 1). For miRNA, this endogenous gene regulator is processed from primary miRNA (pri-miRNA) transcripts of non-coding regions or introns of protein-coding polymerase II transcripts. They are processed by RNase III Drosha to produce approximately 70-nt long pre-miRNAs, which are transported into cytoplasm by exportin-5 and are cleaved by Dicer to become the functional miRNA. Similar to siRNA, they also form a RISC with Argonaut proteins (having RNase $\mathrm{H}$ activity) and bind to their target mRNAs. The modes of actions of siRNA and miRNA depend on the degree of complementation between the siRNA or miRNA and their target sequences. siRNAs usually target coding regions by complementary base-paring and induce sequence-specific cleavage of mRNA substrate [53]; however, miRNAs preferentially recognize target sequences in the 3' UTR of mRNAs and these target sites are often in multi-copy [54-57]. The binding of the miRNAs often takes place with an incomplete base-pairing, although a perfect base-pairing in the seed region (positions nt 2-8 from 5 end of the antisense strand) of miRNA forms the core of interaction. Depending on the complete or partial complementarities between the miRNA and mRNA, the outcome can be cleavage of the target mRNA or repression of translation (Figure. 1) [58, 59].

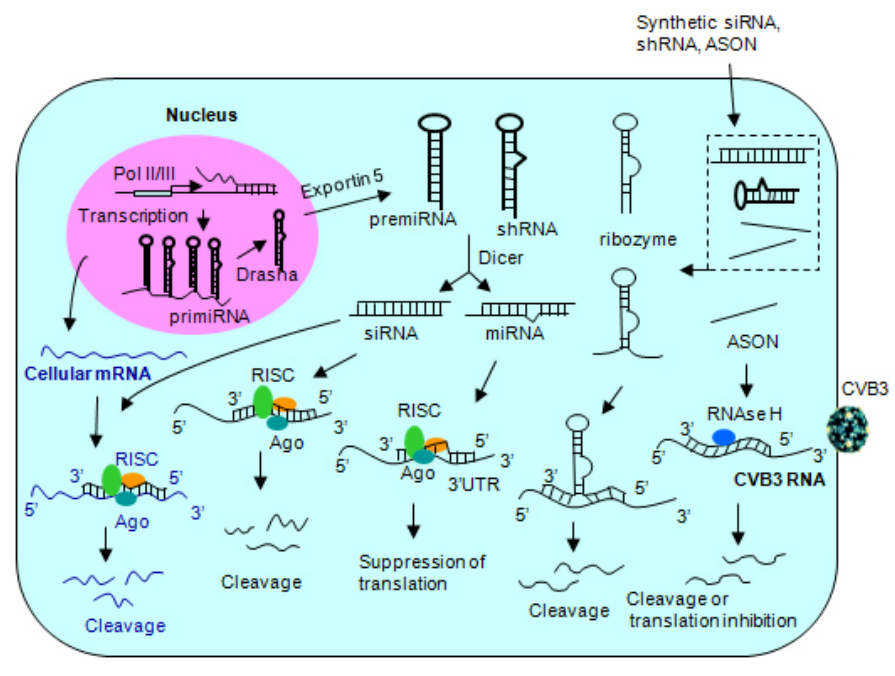

Figure 1. NA-based antiviral strategies to treat viral myocarditis. Antiviral nucleic acid molecules can either be transfected into cells or expressed intracellularly. ASONs hybridize to viral mRNA to induce RNase H-mediated cleavage of RNA strand of the DNA-RNA duplexes. Some modified ASONs cannot induce RNase $\mathrm{H}$ but they have a high affinity for the target and inhibit translation by steric hindrance of ribosome. Binding of ribozymes to the target sequence can trigger cleavage of the viral RNA. siRNAs incorporated in the RISC target the viral RNA by perfect sequence complementation and induce cleavage of the target sequence by RNAse $\mathrm{H}$ activity of Ago protein. miRNAs (or AmiRNAs) target viral RNA by imperfect sequence complementation and induce gene silencing by destabilizing mRNAs and suppression of translation. In addition, siRNAs can also target cellular genes (e.g., viral receptor and signal molecules) involved in viral entry and replication. 
RNAi-mediated antiviral strategies can achieve much higher efficiency than ASONs. Thus, recent studies have focused on the design and evaluation of anti-CVB3 siRNAs. This group of small double-stranded RNAs, as a silencer of target gene expression, can virtually inhibit any genes of virus and cell if the site of targeting within the gene is unique. Thus, the target search for anti-CVB3 siRNAs is not only concentrating on CVB3 genome but also extending to the host cellular genes required for viral infection or replication.

\subsubsection{Targeting the CVB3 genome}

CVB3 genome harbors many cis-acting sequence elements for viral transcription and translation, such as the $5^{\prime}$ and $3^{\prime}$ UTRs, IRES, and other segments for binding of transcription and translation initiation factors. In addition, the viral genome also encodes many essential enzymes for CVB3 multiplication, such as proteases $2 \mathrm{~A}$ and $3 \mathrm{C}$ as well as the RNA-dependent RNA polymerase 3D. These structures are rational targets for design of anti-CVB3 siRNAs. This hypothesis has been tested by a number of groups. The earlier selection of the siRNA targets was focused on CVB3 protease 2A. Almost at the same time, two groups independently found that inhibition of 2A protease by specific siRNAs significantly reduced CVB3 replication. Our laboratory evaluated five siRNAs targeting the 5' UTR, AUG start codon, VP1, 2A and 3D, respectively and found that the siRNA targeting 2A (nts 3543-3561) showed strongest anti-CVB3 activity in HeLa cells, resulting in $92 \%$ reduction of viral replication and siRNAs targeting VP1, 3D and the 5'UTR showed modest antiviral effects, respectively. By mutational analysis of the mechanism of siRNA action, we further found that siRNA functions by targeting the positive strand of the virus and require a perfect sequence match in the central region of the target, but mismatches were more tolerated near the $3^{\prime}$ end than the $5^{\prime}$ end of the antisense strand [60]. This finding on the targeting of siRNA to positive strand of CVB3 was further supported by a later study using siRNA targeting the CVB3 3D gene [61]. We later also conjugated the siRNA-2A with folate to achieve specific delivery of the drug into HeLa cells and inhibited CVB3 replication[62].The second group that studied the siRNA targeting CVB3 2A by Merl and co-workers evaluated antiviral activity of siRNA-2A (nts 3637-3657) in vitro and in highly susceptible type I interferon receptor-knockout mice. They found that siRNA-2A led to a significant reduction of viral tissue titers, attenuated tissue injury and prolonged survival of mice [63]. It is very interesting to point out that although the two groups used different targeting sequences within the 2A RNA, they all achieved high efficiency of antiviral effects. However, the later work by Racchi et al., which used these two siRNAs together to transfect HeLa cells and then infect with CVB3 did not potentiate the anti-CVB3 effect compared with an equimolar concentration of either siRNA [64].

CVB3 RNA polymerase 3D is probably the most frequently used target for design of antiCVB3 siRNAs as it is the only viral enzyme involved in CVB3 RNA replication. To date, at least a half dozen of studies on $3 \mathrm{D}$ have been reported. The earlier in vitro investigations used either un-modified or LNA-modified siRNAs or plasmid vector-expressed shRNAs 
and all achieved significant reduction of viral replication in CVB3-infected HeLa or Cos-7 cells [60, 61, 65-67]. The in vivo evaluation using mouse models also showed very promising results. One study employing transient transfection for in vivo mouse models demonstrated that two of the six candidate siRNAs targeting 3D and VP1, respectively, exerted strong anti-CVB3 effects in viral replication, accompanied by attenuated pancreatic tissue damage [68]. Another in vivo study is the intravenous treatment of mice with an adeno-associated virus vector (AAV2.9) expressing a shRNA targeting 3D [69]. Intravenous injection of recombinant AAV2.9 significantly attenuated cardiac dysfunction compared to vector-treated control mice on day 1 after CVB3 infection. Recently, a study by combination of soluble CAR receptor (sCAR-Fc) and siRNA targeting $3 \mathrm{D}$ achieved a synergistic effect in antiviral effect in human myocardial fibroblast cell culture [14].

Other less frequently used CVB3 target genes are protease 3C, structural protein VP1 and non-structural protein $2 \mathrm{C}$. Like protease $2 \mathrm{~A}$, protease $3 \mathrm{C}$ also plays an important role in the viral life cycle by processing CVB3 polyproteins to generate mature individual structural and non-structural proteins after initial cleavage by $2 \mathrm{~A}[70,71]$. One study designed three siRNAs targeting genes encoding 3C, 2A and 3D of CVB4. Evaluation by transfection of rhabdomyosarcoma (RD) cells demonstrated that siRNA-3C was the most potent siRNA among these three in inhibition of CVB4 replication. This antiviral activity was followed by siRNAs targeting 3D and 2A [72]. The difference in efficiency of these siRNAs was discussed by these authors and they proposed that this may be due to differences in function of these viral enzymes, which are encoded by these regions. The $3 \mathrm{C}$ region encodes a protease $3 C$ which is responsible for the majority of cleavage of the viral polyprotein [71] and $3 \mathrm{C}$ as well as its precursor $3 \mathrm{CD}$ also plays an important role at the level of viral transcription [73]. Protease $3 \mathrm{C}$ has been shown to be critical for interaction with the cloverleaf structures found at the $5^{\prime}$ UTR of the viral genome to deliver the $3 \mathrm{D}$ to the replication complex [74]. They also indicated that since the function of $3 \mathrm{C}$ is required prior to $3 \mathrm{D}$, a down-regulation in $3 \mathrm{C}$ would have a detrimental effect on viral transcription, as available 3D would not be able to carry out replication of CVB4 replication without the assistance of $3 \mathrm{C}$. The authors' interpretation seems to be reasonable; however, according to the order (timing) of action for these enzymes, 2A cleaves the polyprotein prior to $3 \mathrm{C}$ cleavage. For this situation, it may be difficult to explain why the siRNAs targeting 2A did not achieve a more efficacious anti-CVB3 activity than siRNA targeting 3C. Obviously, many issues relating to the mechanisms of action need to be further studied. However, according to the present reports, one point is clear that $2 \mathrm{~A}, 3 \mathrm{C}$ and 3D are three important targets for design anti-CVB3 siRNAs.

Viral structural protein VP1 was also a selected target for testing anti-CVB3 siRNAs; however, data from literature often showed less effectiveness of the siRNA targeting this structural gene compared to that targeting other genes $[60,65,68]$. Due to the absence of a proof-reading activity in $3 \mathrm{D}$, the mutation rate for RNA viruses is as high as $10^{-3}-10^{-4}$ [75]. Thus, in recent years, the discovery of the occurrence of escape mutants due to siRNA treatment of $\mathrm{HCV}$, poliovirus and HIV infections [76-78] greatly encouraged re- 
searchers to search for new approaches to counteract drug resistance. One direction is the application of multiple distinct siRNAs or a siRNA pool to target more than one target genes of the virus [79, 80]. The other direction is the identification of conserved cisacting replication elements (CRE) [81]. Theoretically, the $5^{\prime}$ and $3^{\prime}$ UTRs are the ideal target regions for siRNAs as they harbor a number of conserved cis-acting elements. However, studies with poliovirus and CVB3 found that siRNA residing in these regions are less efficient than siRNAs targeting other regions (e.g., the coding region and particularly the non-structural coding region) in inducing antiviral activity [60, 77, 79, 82]. This low antiviral potency seems to be due to the highly ordered structure of the UTRs itself, as well as to the formation of the protein-RNA complexes in the region, which may block the access of the RISC complexes to its target sequences. To address this issue, Lee and coworkers selected a CRE within the coding region of 2C. Evaluation in HeLa cells demonstrated the down regulation of virus replication and attenuation of cytotoxicity in various strains and human isolates. Cells treated with this siRNA were resistant to the occurrence of viable escape mutants and showed sustained antiviral ability [83]. Based on this study, a similar experiment using siRNA targeting CRE of CVA24 2C was conducted and the authors reported similar observations [84]. These findings from in vitro studies were further strengthened by in vivo evaluation, in which recombinant lentivirus was employed to express shRNAs targeting the CRE of CVB3 2C. Mice injected intraperitoneally with recombinant lentiviruses had significant reductions in viral titers, viral myocarditis and proinflammatory cytokines as well as improved survival rate, after being challenged with CVB3 [85]. Recently, this CRE was further confirmed for a number of enteroviruses, by using a novel program and in vitro evaluation [86].

\subsubsection{Targeting host cellular genes}

Another approach to fight drug resistance caused by escape mutants is the selection of therapeutic targets within the host cellular genes that are involved in virus entry or viral replication. In this regard, the CAR receptor which is shared by CVB3 and adenovirus is an attractive candidate since both CVB3 and adenovirus are considered as the common causal agents of myocarditis. To date, two studies have been reported to silence CAR expression with specific siRNAs. One study reported that transfection of HeLa cells with siRNAs, siCAR2 or siCAR9, almost completely silenced the expression of CAR and that further analysis by viral plaque assay revealed $\sim 60 \%$ reduction of CVB3 particle formation [67]. Another study, using cardiac-derived HL-1 cell line and primary neonatal cardiomyocytes (PNCMs) demonstrated that treatment with recombinant adenoviruses expressing shRNAs against CAR resulted in almost completely silencing of CAR expression in both HL-1 cells and PNCMs. CAR knockout resulted in inhibition of CVB3 infections by up to $97 \%$ in HL-1 and up to $90 \%$ in PNCMs. Adenoviruses were inhibited by only $75 \%$ in HL-1, but up to $92 \%$ in PNCMs [87].

Another host gene, the tissue inhibitor of matrix metalloproteinase-1 (TIMP-1), has been suggested to be a potential target for siRNA to ameliorate CVB3-induced myocarditis. 
This suggestion is based on the investigation of Crocker and colleagues on a new role of TIMP-1 in exacerbating CVB-induced myocarditis. They found that TIMP-1 expression was induced in the myocardium by CVB3 infection. Surprisingly, TIMP-1 knockout mice exhibited a profound attenuation of myocarditis, with increased survival. The amelioration of disease in TIMP-1 knockout mice was not attributable to either an altered T-cell response to the virus nor to reduced viral replication. These data allowed the authors to propose and prove a novel function for TIMP-1. Its highly localized up-regulation might arrest the matrix metalloproteinase (MMP)-dependent migration of inflammatory cells at the sites of infection, thereby anatomically focusing the adaptive immune response. Finally, the benefits of TIMP-1 blockage in treating CVB3-induced myocarditis were confirmed by administration of siRNAs targeting TIMP-1, which diminished the disease. However, this improvement of the treatment is not due to changes of viral titers, as demonstrated by viral plaque assay [88].

Recently, active investigations on CVB3-induced signal transduction pathways have provided new avenues for the search of therapeutic targets for the treatment of myocarditis. Since CVB3, like other picornaviruses, requires the activation of certain signal pathways for initiating their life cycle, inactivation of some signal molecules in the signal cascade with specific siRNAs would block CVB3 replication. Such kind of studies that have been documented thus far include i) the knockdown of ubiquitin expression by siRNAs to down-regulate the ubiquitination and subsequent alteration of protein function and/or protein degradation [89]; ii) silencing of proteosome activator REG to inhibit the REGmediated degradation of several important intracellular proteins [90], such as cyclin-dependent kinase inhibitors p21, p16 and tumor suppressor p53; and iii) knockdown of genes critical for autophagy formation including ATG7, Beeclin-1 and VPS34 [91]. Although these target genes mentioned above have been tested in vitro using specific siRNAs in signal transduction studies and showed promising outcomes, their potential serving as a therapeutic target for treatment of CVB3 infection needs further evaluation by pharmacological study in animal models.

\subsubsection{Anti-CVB3 artificial miRNAs}

miRNAs are a group of recently discovered new regulators of gene expression. These endogenous regulators control one third of human gene expression [92, 93]. Thus, endogenous miRNAs are important targets for gene therapy and artificial miRNAs (AmiRNA) are useful tools for inhibiting disease-causing gene expression [94, 95], which have been tested in numerous studies on the treatment of cancers, cardiovascular diseases, genetic diseases and other viral infections. To test its anti-CVB3 effect, we constructed three short hairpin AmiRNAs (AmiR-1, -2 and -3) targeting the stem-loop of the 3' UTR of CVB3 with mismatches at the middle region of the target [96]. Transfection of HeLa cells showed over-expression of these mature AmiRNAs as determined by real time quantitative RT-PCR. After these AmiRNA-expressing cells were infected with CVB3, the viral 
titers were reduced $\sim 10$ folds in cell cultures treated with AmiR-1 or AmiR-2 but not in those treated with AmiR-3, at $24 \mathrm{~h}$ post infection. Mutational analysis of the targeting sites of AmiRNAs demonstrated that the central region but not the seed region of AmiRNAs is more tolerant to target mutation. In this study we also performed targeted delivery of the AmiRNAs to host cells through ligand-receptor interactions. Recently, another group evaluated the antiviral activity of miR-342-5p in CVB3 infection of tissue culture cells. They found that miR-342-5p functions by targeting CVB3 $2 \mathrm{C}$ region at nts 4989-5010, which is conserved in CVB type 1-5. Treatment of HeLa cells by transfection significantly inhibited viral RNA and protein synthesis. Mutation of the target site or using inhibitor of miR-342-5p decreased the antiviral effect in vitro [97].

In summary, the NA-based antivirals against CVB3 infection discussed above have shown great promise thus far (Table 1); however, none of them has reached the step for clinical trial. Many limitations such as drug stability, toxicity and targeted delivery need to be overcome before its addition to the list of clinical application.

\section{Immunomodulatory therapy}

As discussed above, the effectiveness of immunosuppressive therapy for viral myocarditis is controversial; we here focus the immunomodulatory therapy on immunoglobulin (Igs) treatment and immunoadsorption.

\subsection{Immunoglobulin treatment}

IgGs have already been shown to be efficacious treatments for Kawasaki disease [98], idiopathic thrombocytopenic purpura, and numerous neuroimmunologic disorders including Guillain-Barre syndrome [99]. The rationale to use IgG in viral infections results from their antiviral and immunomodulating effects. In the setting of viral myocarditis, IgGs can be utilized to suppress superfluous immune activation which may include an autoimmune component, but such treatment has shown conflicting results. IgGs prevented myocardial injury in experimental models of myocarditis [100, 101]. Even when administered in a delayed manner, IgG administration was able to limit scar formation and improve left ventricular (LV) function [101] or reduced pro-inflammatory TNF- $\alpha$ coupled with increased anti-inflammatory interleukins-1 and -10 [102]. More recently, Kishimoto et al [103] also showed improved heart function in adults with myocarditis and DCM. The same group recently showed that immunoglobulin treatment ameliorates myocardial injury in experimental autoimmune myocarditis associated with suppression of reactive oxygen species [104]. To date, however, there has only been one randomized clinical trial investigating IgG treatment in patients with myocarditis. McNamara et al [105] showed that, in a placebo-controlled prospective trial in patients with recent-onset DCM and myocarditis, intravenous immunoglobulin administration did not improve LV function. 


\begin{tabular}{|c|c|c|c|c|}
\hline Category & Target & Model system & Delivery route & Reference \\
\hline PS-ASON & $\begin{array}{l}5^{\prime} \& 3^{\prime} U T R s, \text { IRES, start } \\
\text { codon }\end{array}$ & HeLa cell, mice & Transfection & Wang 2001 (36) \\
\hline PS-ASON & 3'end of CVB3 & HL-1 cells, mice & $\begin{array}{l}\text { Transfection, IV } \\
\text { injection }\end{array}$ & Yuan 2004 (37) \\
\hline MOP-ASON & $\begin{array}{l}\text { 5' \& 3'UTRs, IRES, start } \\
\text { codon, minus strand }\end{array}$ & HeLa, HL-1 cell, mice & $\begin{array}{l}\text { Transfection, IV } \\
\text { injection }\end{array}$ & Yuan 2006 (38) \\
\hline $\begin{array}{l}\text { CpG } \\
\text { oligomer }\end{array}$ & no & PBMCs & Treatment & Cong 2007 (39) \\
\hline siRNA & 2A, VP1, 3D & HeLa cells & Transfection & Yuan 2005 (60) \\
\hline siRNA & $2 \mathrm{~A}$ & HeLa cells & pRNA vector & Zhang 2009 (62) \\
\hline siRNA & $2 \mathrm{~A}$ & HeLa cells, mice & $\begin{array}{l}\text { Hydrodynamic } \\
\text { Transfection }\end{array}$ & Merl 2005 (63) \\
\hline siRNA & $2 \mathrm{~A}$ & HeLa cells & Transfection & Racchi 2009 (64) \\
\hline shRNA & $3 D$ & HeLa cells & $\begin{array}{l}\text { Transfection of } \\
\text { double expression } \\
\text { plasmid }\end{array}$ & Schubert 2005 (66) \\
\hline SiRNA & 3D, VP1 & HeLa cells & Transfection & Ahn 2005 (65) \\
\hline LNA-siRNA & $3 D$ & Cos-7 cells & Transfection & Schubert 2007 (61) \\
\hline siRNA & siRNA pool & LLC-MK2 cells & Transfection & Nygardas 2009 (80) \\
\hline shRNA & VP1, 3D, 5' \& 3'UTR & Cos-7 cells, mice & $\begin{array}{l}\text { Hydrodynamic } \\
\text { Transfection }\end{array}$ & Kim J-Y 2008 (85) \\
\hline $\begin{array}{l}\text { siRNA \& } \\
\text { sCAR-Fc }\end{array}$ & $3 D$ & HMF & Transfection & Werk D 2009 (14) \\
\hline shRNA & $3 D$ & HeLa, PNCMs, mice & $\begin{array}{l}\text { Transduction, IV, AAV } \\
\text { vector }\end{array}$ & Fechner 2008 (69) \\
\hline siRNA (CVB4) & $3 \mathrm{D}, 3 \mathrm{C}, 2 \mathrm{~A}$ & RD cells & Transfection & Tan , $2010(72)$ \\
\hline shRNA & $2 C$ & Mice & $\begin{array}{l}\text { IP injection, lentivirus } \\
\text { vector }\end{array}$ & Lee 2007 (83) \\
\hline $\begin{array}{l}\text { shRNA } \\
\text { (CVA24) }\end{array}$ & $2 C$ & HeLa, HCC & $\begin{array}{l}\text { Transfection of } \\
\text { plasmid }\end{array}$ & Jun 2008 (84) \\
\hline $\begin{array}{l}\text { siRNA } \\
\text { (entero- } \\
\text { viruses) }\end{array}$ & $2 C$ & HeLa, Vero cells & Transfection & Lee 2009 (86) \\
\hline shRNA & CAR & HL-1, PNCMs & Adenovirus vector & Fechner 2007 (87) \\
\hline siRNA & TIMP-1 & Mice & IV injection & Crocker 2007 (88) \\
\hline siRNA & CAR, 3D & HeLa,Cos-7 cells & Transfection & Werk 2005 (67) \\
\hline siRNA & Ubiquitin & HeLa cells & Transfection & Si 2008 (89) \\
\hline siRNA & ATG7, Beclin-, VPS34 & HeLa cells & Transfection & Wong 2008 (91) \\
\hline siRNA & $\begin{array}{l}\text { Proteasome activator } \\
\text { REGY }\end{array}$ & HeLa cells & Transfection & Gao G 2010 (90) \\
\hline
\end{tabular}

Table 1. NA-based agents for the treatment of CVB3 infection 


\subsection{Immunoadsorption}

The rationale for immunoadsorption is to lower concentration of cardiotoxic antibodies in patients plasma, and with serial treatments over 5 or more days, extract antibodies and immune complexes from the heart as well [106]. There is evidence that removal of circulating antibodies against cardiac proteins by immunoadsorption in DCM improved cardiac function [107] and reduced clinical and humoral markers of heart failure severity [108, 109] as well as improved hemodynamic parameters [110]. Further immunoadsorption decreased myocardial inflammation. In patients with inflammatory cardiomyopathy, LV systolic function improved after protein A immunoadsorption [111]. Recently, Nagatomo et al reported that immunoadsorption using IgG3-specific tryptophan column for patients with refractory heart failure due to DCM is a safe treatment and has shown short term efficacy. Long term follow-up is needed to confirm the effects on cardiac function and on morbidity/mortality in such patients [112]. Another recent study demonstrated that immunoadsorption treatment improved endothelial function in patients with chronic inflammatory DCM. This effect is associated with a significant drop in circulating microparticles [113].

\section{Antiviral treatment}

\subsection{Compounds inhibiting viral replication}

As mentioned earlier, ribavirin is a frequently used antiviral agent. This agent is a nucleoside analogue and can block viral transcription elongation and thus can be used to inhibit a number of RNA viral infections, including CVB3 [114, 115]. Recently, new antiviral compounds have been synthesized. Harki et al. synthesized some cytidine analogues and one of them, 5-nitrocytidine, decreased CVB3 titer in infected cells, with 12fold higher efficiency than ribavirin, but so far the in vivo evaluation has not been reported [116]. Other strategies for antiviral compound design are inhibitors of viral protease, RNA-dependent RNA polymerase or other nonstructural proteins, such as guanidine hypochloride, HBB, MRL-1237 and TBZE-02, which interact with viral 2C protein resulting in inhibition of viral RNA transcription.

Nitrooxide (NO) donor is another form of antiviral agents interfering with viral nonstructural proteins. They inhibit enterovirus proteases 2A and 3C [109, 117]. The NO donors nitroglycerin (GTN) and isosorbide dinitrate (ISDN) can suppress CVB3 replication by inhibiting viral proteases in vitro. Further, in vivo study showed that GTN significantly reduced myocarditis after administration by decreasing immune cell infiltration and tissue fibrosis up to 14 day post infection [111]. In another study using a CVB3 myocarditis mouse model, treatment with NO-metoprolol showed enhanced therapeutic benefit compared to metoprolol, with significant reduction of viral RNA synthesis, body weight loss, infiltration and fibrosis score [118]. Interestingly, another study using cinnamaldehyde, which can reduce plasma nitric oxide (NO) content, also showed the effectiveness in treatment of CVB3 myocarditis. This compound also reduced NF- $\kappa B$, inducible nitric oxide synthase and TLR4 expression. Thus, the underlying mechanism is likely by inhibiting the TLR4-NF- $\kappa B$ signal transduction 
pathway [119]. Recently, a protein-based CVB3 protease 3C inhibitor, 3CPI, demonstrated that treatment by way of a micro-osmotic pump delivery significantly inhibited viral proliferation, and attenuated myocardial inflammations, subsequent fibrosis, and CVB3-induced mortality in vivo [120].

\subsection{Interferons (IFNs)}

IFNs are critical cytokines of the innate immune response released in response to stimuli with particular importance in viral infection. IFN signal through one of two receptor groups which dictates their subtype IFN-alpha and IFN-beta are of type I and IFN-gamma is of type II. Type I IFNs trigger critical antiviral responses whereas, type II IFNs contribute to immune enhancement and modulation including an important role in macrophage activation. The best studied of these proteins is the IFN-gamma. Infections in IFN-gamma-deficient mice showed that IFN-gamma triggers release of IL-1, IL-4 and transforming growth factor, the latter being sentinel to development of cardiac fibrosis [121]. Notably, expression of IFNgamma by IFN-gamma recombinant CVB3 vector protected mice against infection of lethal CVB3H3 variant by decreasing the viral load and spread as well as tissue destruction when given prior to or directly after viral infection [122, 123].

Type I IFNs have also shown promise in the treatment of viral myocarditis. IFN-alpha is known to trigger a number of biological cascades to inhibit virus infection. IFN-alpha was used successfully to treat two patients with acute enterovirus-induced myocarditis [123]. As well, IFN-beta therapy has been used to improve the prognosis for patients with DCM [124]. Recently, experimental evidence has suggested that IFN-beta can also be used as an antiviral treatment and can improve outcome in viral myocarditis [125, 126]. These studies showed that treatment with IFN-beta resulted in an elimination of cardiac viral load, protected cardiomyocytes against injury and decreased inflammatory cell infiltrates. In a placebo controlled, randomized, double-blind, phase II trial (BICCstudy), 143 patients with inflammatory DCM and viral myocarditis were treated with IFN-beta-1b and showed significant reduction of viral load (enterovirus) in myocardium; however, complete viral elimination (parvovirus B19) was not achieved in all patients [127]. This is probably due to that this virus responds less well upon IFN-beta treatment. Novel IFN amplification using poly(inosinic acid)-poly(cytidylic acid) [poly(IC)], IFN-alpha-2b, pegylated IFN-alpha-2b (PEG-INTRON-alpha-2b), and ampligen have proved successful in blocking virus infection [128]. Oral administration of IFN-alpha-2b expressing bacteria (B. longum) also protects mice against CVB3-induced myocarditis [129]. In addition, type I interferons induced by modified 3p-siRNA specifically targeting CVB3 genome significantly reduced viral load and damage of the heart [130].

\subsection{Soluble receptor analogues}

Another similar strategy in developing antiviral agents is to block viral entry by utilization of recombinant soluble protein of CAR receptor. Detailed review can be found in a recent article [21]. Soluble receptor analogues bind to the virus before the viral binding to its receptor, thus preventing binding of virus and subsequent entry to the target cells. Several re- 
search groups designed and produced this type of analogues by recombinant DNA technology to increase its efficiency. The most common strategy is the modification of the protein by fusion of virus binding domain on the receptor, CAR or DAF, with the C-terminus of the human IgG1 Fc region, resulting in a dimeric antibody-like molecule. This modification greatly enhanced the solubility and stability of the fusion protein [13, 15, 131-133]; as well as increased the efficiency in viral neutralization [134]. However, one study reported the possible side effects caused by this approach, which demonstrated that after treatment with recombinant CAR4/7, animal showed aggravated myocardium inflammation, tissue damage and presence of CAR-specific antibody. The possible mechanism leading to this problem may be due to the bacteria-produced recombinant protein altered the glycosylation pattern and increased the immunogenicity [135]. Recently, another study simultaneously applied soluble CAR-Fc and siRNA targeting CVB3 genome exerted synergistic antiviral activity in the treatment of a persistently infected cardiac cell line in vitro [14].

\section{Natural products}

Natural products occupy tremendous chemical structural space - unmatched by any other small molecule families - possess a range of biological activities, remain the best sources of drugs and drug leads, and serve as outstanding small molecule probes for dissecting fundamental biological processes [136, 137]. Natural products are evolutionarily optimized to be drug-like. They are generally more potent and specific than synthetic molecules, suggesting increased binding affinities for their cognate protein receptors. This characteristic may be attributed to the fact that natural products are biosynthetically made through repeated interaction with modulating enzymes; thus their ability to interact with biological macromolecules is intrinsic to their structures. In addition, they may result from a complex evolutionary interaction between co-occupants of an ecological niche, resulting in the optimization of natural products in a process that is inaccessible to synthetic compounds [138-140].

The natural products, such as Astragalus membranaceus, Salviae miltiorrhizae, Sophorae flavescentis and Phyllanthus emblica or Chinese proprietary medicines, such as Shenmai, Shuanghuangkian and Qishaowuwei, have been long known to be effective in treating viral myocarditis. However, the components of the medicine and the mode of action are largely unknown [141]. Recent years, emerging studies focused on the isolation of the major component of the medicine and the mechanisms of action. Astragaloside IV is probably the most studied natural compound in anti-myocarditis caused by viral infection. Two groups isolated this compound from Astragalus membranaceus and Radix Astragali respectively and all showed the effectiveness of this component in treatment of CVB3 infection of the heart. One group demonstrated that treatment could significantly decrease virus load, mononuclear cell infiltration and cardiomyocyte injury in mice. They further found that astragaloside IV exerted antiviral effects against CVB3 by upregulating IFN-gamma expression [142]. The other group showed that astragalus treatment significantly decreased the fibrosis of the heart tissue and increased the mouse survival rate; further analysis revealed that this cardio- 
protective effect is largely due to the inhibition of the TGF-beta 1-Smad signaling in DCM [143]. Sophoridine, an alkaloid extracted from Sophora flavescens, has been evaluated in mice and rats. The results showed that sophoridine treatment obviously decreased viral titer and enhanced mRNA expression of IL-1 and IFN-gamma but decreased TNF-alpha. They concluded that sophoridine itself but not its metabolites is responsible for its antiviral activity by regulating cytokine expression [144]. Recently, another natural product phyllaemblicin B, the main sesquiterpenoid glyside isolated from roots of Phyllanthus emblica, was reported to reduce CVB3-iduced apoptosis both in vitro and in vivo. In CVB3 myocarditis mouse model, this compound reduced CVB3 titer, decreased activities of LDH and CK in murine serum, and alleviated pathological damage of the myocardium [145].

\section{Cellular cardiomyoplasty}

The critical loss of functional cardiomyocytes causes a severe deterioration of contractility, which eventually results in heart failure. To reverse the myocardial injuries in disease progression, the damaged, hypocontractile and necrotic myocytes need be replaced. Although in contrast to the long-standing dogma that mammalian heart loses capability of proliferation in injuries after birth, there is much evidence now to support a degree of regeneration in postnatal human heart. Regardless of whether the proliferating myocytes are derived from the resident cardiomyocytes or circulating stem cells, it is obvious that this self-renew mechanism is not sufficient in amount to prevent or block the heart failure.

Cellular cardiomyoplasty (CCM) is now emerging as one of the most promising therapeutic techniques for the augmentation and regeneration of injured myocardium [146]. The strategy is to introduce less differentiated or undifferentiated cells, or in vitro derived cardiomyocytes into injured heart to mediate repair of chronically injured myocardium [147]. Cells of various origins and stages of differentiation, but with the capability of differentiating into a contractile phenotype have been utilized. The most frequently referred cell types for such treatment are skeletal myoblast, embryonic stem cells, and bone marrow cells which contain lineages of hematopoietic and mesenchymal stem cells [148].

All transplanted cell lines mentioned above showed some improvements on myocardial regional and/or global function in a variety of animal models and some have been investigated in clinical trials. Although the mechanism of improved cardiac function with implanted cells requires further study, the following evidence may help us to understand the general therapeutic process: i) systolic contraction generated by implanted cardiomyocytes; ii) alteration and attenuation of deleterious ventricular remodeling; iii) induction of angiogenesis by released growth factors such as vascular endothelial growth factor, basic fibroblast growth factor, and angiopoietin-1.

To date, a number of studies have been conducted for the treatment of myocardial infarction or chromic myocardial ischemia, only a few experimental cell-based studies are directed at treating nonischemic cardiomyopathy $[149,150]$. The treatment studies for virus-induced viral myocarditis or DCM is even fewer. Here we only found two reports 
on the CVB3-induced myocarditis. The pioneer work by van Linthout and co-workers demonstrated that mesenchymal stem cells (MSCs) are potential therapeutic cells for the CVB3-viral myocarditis [151]. This finding is largely based on that these cells express a low level of CAR receptor and thus are not sensitive to CVB3 infection. In co-culture experiments with the cardiomyocytes HL-1, MSCs reduced CVB3-induced cell apoptosis and oxidative stress. Furthermore, MSCs diminished viral progeny release by approximately 5-fold. Importantly, intravenous injection of MSCs decreased cardiac apoptosis and improved LV function in a murine CVB3 myocarditis model. A detailed study on the mechanism revealed that the protective effect of MSCs is mediated in an NO-dependent manner and requires priming via IFN-gamma. Another recent study using cardiac-derived adherent proliferating cells (CAPs) showed similar results as that using MSCs [152]. CAPs only minimally express both CAR and DAF receptors, which translates to minimal CVB3 copy numbers, and without viral particle release after infection. Co-culture of CAPs with CVB3-infected HL-1 cells resulted in a reduction of CVB3-induced HL-1 cell apoptosis and viral progeny release. In addition, CAPs have immunomodulatory feature and can lead to a decrease in CVB3 load, myocyte death and an improvement in LV contractility parameters in murine acute CVB3 myocarditis. CAPs exert protective effects in an NO-and IL-10-dependnet manner and require IFN-gamma for their activation.

Despite many questions regarding stem cell plasticity have not been answered, exploratory clinical trials are currently underway with both skeletal myoblasts [153, 154] and bone marrow-derived cells $[155,156]$. It is estimated that more than 15 patients have been treated with CCM worldwide, and the number of patients treated with autologous skeletal myoblasts is equivalent to those treated with bone marrow cells [156]. These preliminary results of CCM are encouraging. However, the potential for this treatment will heavily depend on conducting more rigorously controlled and randomized clinical trials with appropriate endpoints to show a clear therapeutic benefit of this approach. In addition, for CCM to become a widely accepted therapy in the future, fundamental questions such as best cell source, appropriate cell dose, timing of implantation, optimum delivery mode, mechanism of action, electrical and mechanical integration, cell survival and long term fate of transplanted cells, need to be addressed.

\section{Concluding remarks}

Since the last decades, a number of new strategies have been emerged in drug development for treatment of viral myocarditis and its sequela DCM, which are summarized in Figure 2. As myocarditis can be induced by a number of viruses, rapid and timely pathogen identification is critically important for guiding early and targeted treatments. Certainly, rapid, sensitive and specific detection of a particular virus or even viral subtype in human samples by detection of virus-specific genes would facilitate targeted treatments. This is particularly crucial for the treatments using nucleic acid-based antiviral agents targeting viral RNA. 


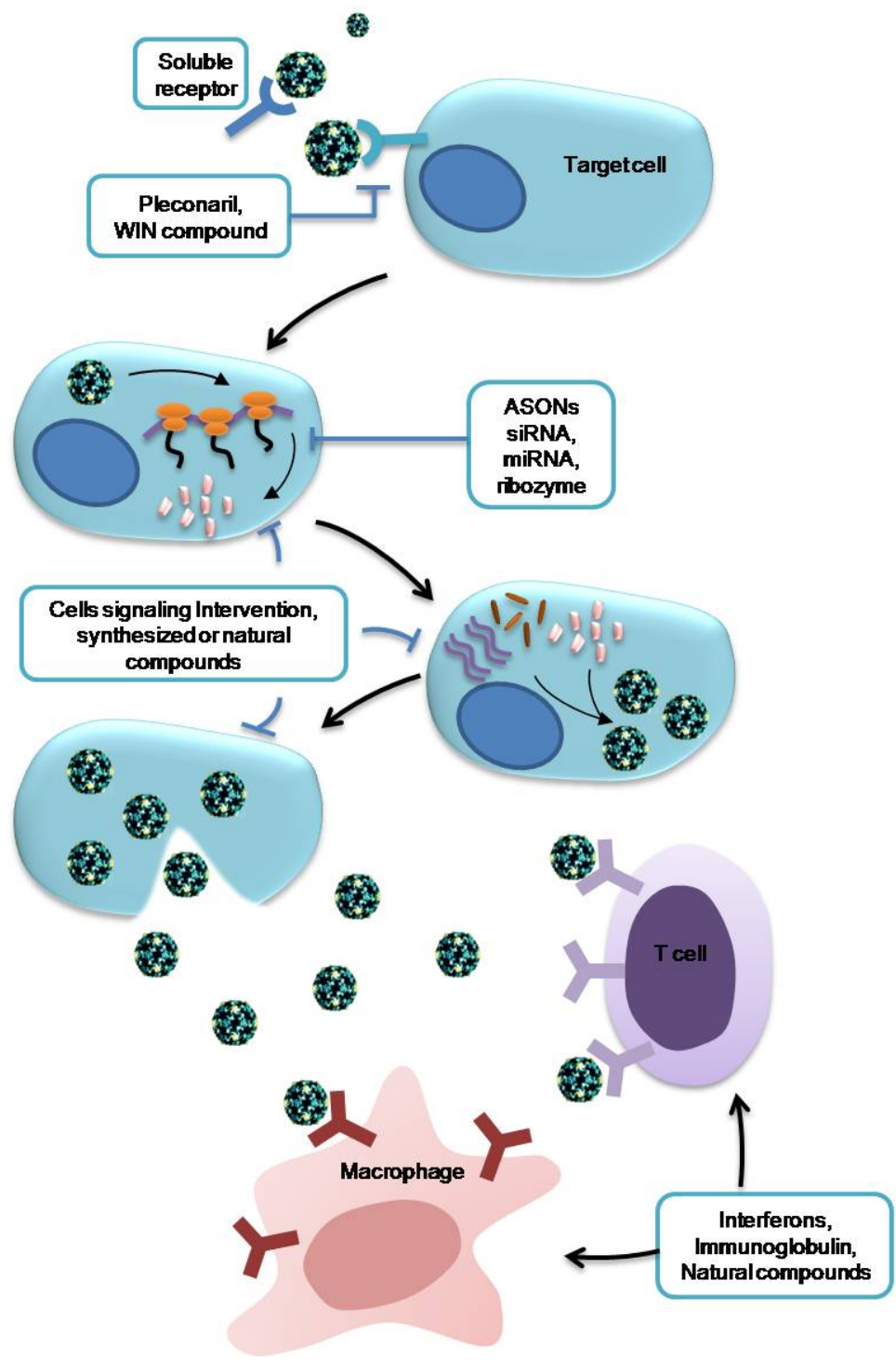

Figure 2. Antiviral strategies for CVB3 infection. The potential therapeutic targets in viral life cycle and the subsequent inflammatory response are indicated for different antiviral agents.

As CVB3 is a RNA virus and has a high mutation rate, drug resistant mutations pose potential obstacles. Therefore, drug targeting on viral proteins for viral replication is another choice for drug design. For example, the inhibition of RNA-dependent RNA polymerase or proteases of CVB3 may offer great promise since their functions are essential for the virus but not for the cell. In the treatment of infection using nucleic acid-based antiviral agents, 
simultaneous application of several drugs may achieve synergistic effects and also reduce the emergence of drug resistance. In addition, combination with the non-nucleic acid-based drugs, such as interferon or soluble receptor, may also achieve the same goal. Recent emerging of the artificial microRNA technology provides another strategy for overcoming drug resistance because miRNA targeting requires partial complementation and is more tolerant to target mutation than siRNA. In searching for new antiviral drugs, although the natural products have long been known to be valuable sources of such agents, progresses in this area of research are not significant as compared to other areas of drug development. Thus more efforts should be made in the screen of the natural antiviral compounds. For end-stage therapy, in light of the preliminary clinical studies, CCM is no doubt an exciting area. We look forward with great anticipation to future clinical studies and a greater understanding of the mechanism of action, which will potentially lead to clinical applications.

\section{Acknowledgements}

The work was supported by a China-Canada (CIHR) Joint Health Research Initiative grant. Xin Ye is supported by a University Graduate Fellowship.

\section{Author details}

Decheng Yang ${ }^{1,2}$, Huifang Mary Zhang ${ }^{1,2}$, Xin Ye ${ }^{1,2}$, Lixin Zhang ${ }^{3}$ and Huanqin Dai ${ }^{3}$

1 Department of Pathology and Laboratory Medicine, University of British Columbia, Canada 2 The Institute for Heart + Lung Health at St. Paul's Hospital, Vancouver, Canada

3 Chinese Academy of Sciences Key Laboratory of Pathogenic Microbiology and Immunology, Institute of Microbiology, Beijing, PRC

\section{References}

[1] Andreoletti, L., et al. Viral causes of human myocarditis. Archive of Cardiovascular Diseases, 2009; 102(6-7) 559-68.

[2] Horwitz, M.S., et al. Transforming growth factor-beta inhibits coxsackievirus-mediated autoimmune myocarditis. Viral Immunology, 2006; 19(4) 722-33.

[3] Blauwet, L.A. and Cooper, L.T. Myocarditis. Progress in Cardiovascular Diseases, 2010; 52(4) 274-88. 
[4] Hosenpud, J.D., et al. The Registry of the International Society for Heart and Lung Transplantation: eighteenth Official Report-2001. Journal of Heart and Lung Transplantation, 2001; 20(8) 805-15.

[5] Drory, Y., et al. Sudden unexpected death in persons less than 40 years of age. American Journal of Cardiology, 1991; 68(13) 1388-92.

[6] Verma, B., Bhattacharyya, S., and Das, S. Polypyrimidine tract-binding protein interacts with coxsackievirus B3 RNA and influences its translation. Journal of General Virology; 91(Pt 5) 1245-55.

[7] Cheung, P., et al. Specific interaction of HeLa cell proteins with coxsackievirus B3 3'UTR: La autoantigen binds the 3' and 5'UTR independently of the poly(A) tail. Cellular Microbiology, 2007; 9(7) 1705-15.

[8] Liu, Z., et al. Structural and functional analysis of the 5' untranslated region of coxsackievirus B3 RNA: In vivo translational and infectivity studies of full-length mutants. Virology, 1999; 265(2) 206-17.

[9] Yang, D., et al. In vitro mutational and inhibitory analysis of the cis-acting translational elements within the 5' untranslated region of coxsackievirus B3: potential targets for antiviral action of antisense oligomers. Virology, 1997; 228(1) 63-73.

[10] Melchers, W.J., et al. Kissing of the two predominant hairpin loops in the coxsackie B virus 3' untranslated region is the essential structural feature of the origin of replication required for negative-strand RNA synthesis. Journal of Virology, 1997; 71(1) 686-96.

[11] Wang, J., et al. Structural requirements of the higher order RNA kissing element in the enteroviral 3'UTR. Nucleic Acids Research, 1999; 27(2) 485-90.

[12] Raschperger, E., et al. The coxsackie- and adenovirus receptor (CAR) is an in vivo marker for epithelial tight junctions, with a potential role in regulating permeability and tissue homeostasis. Experimental Cell Research, 2006; 312(9) 1566-80.

[13] Pinkert, S., et al. Prevention of cardiac dysfunction in acute coxsackievirus B3 cardiomyopathy by inducible expression of a soluble coxsackievirus-adenovirus receptor. Circulation, 2009; 120(23) 2358-66.

[14] Werk, D., et al. Combination of soluble coxsackievirus-adenovirus receptor and anticoxsackievirus siRNAs exerts synergistic antiviral activity against coxsackievirus B3. Antiviral Research, 2009; 83(3) 298-306.

[15] Yanagawa, B., et al. Soluble recombinant coxsackievirus and adenovirus receptor abrogates coxsackievirus b3-mediated pancreatitis and myocarditis in mice. Journal of Infectious Diseases, 2004; 189(8) 1431-9.

[16] Freimuth, P., Philipson, L., and Carson, S.D. The coxsackievirus and adenovirus receptor. Current Topics in Microbiology and Immunology, 2008; 323 67-87. 
[17] Shafren, D.R., Williams, D.T., and Barry, R.D. A decay-accelerating factor-binding strain of coxsackievirus B3 requires the coxsackievirus-adenovirus receptor protein to mediate lytic infection of rhabdomyosarcoma cells. Journal of Virology, 1997; 71(12) 9844-8.

[18] Schultz, J.C., et al. Diagnosis and treatment of viral myocarditis. Mayo Clinic Proceedings, 2009; 84(11) 1001-9.

[19] Dennert, R., Crijns, H.J., and Heymans, S. Acute viral myocarditis. European Heart Journal, 2008; 29(17) 2073-82.

[20] Rose, N.R. Myocarditis: infection versus autoimmunity. Journal of Clinical Immunology, 2009; 29(6) 730-7.

[21] Fechner, H., et al. Pharmacological and biological antiviral therapeutics for cardiac coxsackievirus infections. Molecules, 2011; 16(10) 8475-503.

[22] Kindermann, I., et al. Update on myocarditis. Journal of the American College of Cardiology, 2012; 59(9) 779-92.

[23] Cooper, L.T., et al. The role of endomyocardial biopsy in the management of cardiovascular disease: a scientific statement from the American Heart Association, the American College of Cardiology, and the European Society of Cardiology. Endorsed by the Heart Failure Society of America and the Heart Failure Association of the European Society of Cardiology. Journal of the American College of Cardiology, 2007; 50(19) 1914-31.

[24] Frustaci, A., et al. Immunosuppressive therapy for active lymphocytic myocarditis: virological and immunologic profile of responders versus nonresponders. Circulation, 2003; 107(6) 857-63.

[25] Groarke, J.M. and Pevear, D.C. Attenuated virulence of pleconaril-resistant coxsackievirus B3 variants. Journal of Infectious Diseases, 1999; 179(6) 1538-41.

[26] Kaiser, L., Crump, C.E., and Hayden, F.G. In vitro activity of pleconaril and AG7088 against selected serotypes and clinical isolates of human rhinoviruses. Antiviral Research, 2000; 47(3) 215-20.

[27] Reisdorph, N., et al. Human rhinovirus capsid dynamics is controlled by canyon flexibility. Virology, 2003; 314(1) 34-44.

[28] Fohlman, J., et al. Antiviral treatment with WIN 54954 reduces mortality in murine coxsackievirus B3 myocarditis. Circulation, 1996; 94(9) 2254-9.

[29] Fox, M.P., Otto, M.J., and McKinlay, M.A. Prevention of rhinovirus and poliovirus uncoating by WIN 51711, a new antiviral drug. Antimicrobial Agents and Chemotherapy, 1986; 30(1) 110-6.

[30] Lewis, J.K., et al. Antiviral agent blocks breathing of the common cold virus. Proceedings of the National Academy of Sciences of the United States of America, 1998; 95(12) 6774-8. 
[31] Pevear, D.C., et al. Activity of pleconaril against enteroviruses. Antimicrobial Agents and Chemotherapy, 1999; 43(9) 2109-15.

[32] Schmidtke, M., et al. New pleconaril and [(biphenyloxy)propyl]isoxazole derivatives with substitutions in the central ring exhibit antiviral activity against pleconaril-resistant coxsackievirus B3. Antiviral Research, 2009; 81(1) 56-63.

[33] Stein, D., et al. A specificity comparison of four antisense types: morpholino, 2'-Omethyl RNA, DNA, and phosphorothioate DNA. Antisense and Nucleic Acid Drug Development, 1997; 7(3) 151-7.

[34] Hoke, G.D., et al. Effects of phosphorothioate capping on antisense oligonucleotide stability, hybridization and antiviral efficacy versus herpes simplex virus infection. Nucleic Acids Research, 1991; 19(20) 5743-8.

[35] Cotten, M., et al. 2'-O-methyl, 2'-O-ethyl oligoribonucleotides and phosphorothioate oligodeoxyribonucleotides as inhibitors of the in vitro U7 snRNP-dependent mRNA processing event. Nucleic Acids Research, 1991; 19(10) 2629-35.

[36] Wang, A., et al. Specific inhibition of coxsackievirus B3 translation and replication by phosphorothioate antisense oligodeoxynucleotides. Antimicrobial Agents and Chemotherapy, 2001; 45(4) 1043-52.

[37] Yuan, J., et al. A phosphorothioate antisense oligodeoxynucleotide specifically inhibits coxsackievirus B3 replication in cardiomyocytes and mouse hearts. Laboratory Investigation, 2004; 84(6) 703-14.

[38] Yuan, J., et al. Inhibition of coxsackievirus B3 in cell cultures and in mice by peptideconjugated morpholino oligomers targeting the internal ribosome entry site. Journal of Virology, 2006; 80(23) 11510-9.

[39] Cong, Z., et al. A CpG oligodeoxynucleotide inducing anti-coxsackie B3 virus activity in human peripheral blood mononuclear cells. FEMS Immunology and Medical Microbiology, 2007; 51(1) 26-34.

[40] Peracchi, A. Prospects for antiviral ribozymes and deoxyribozymes. Reviews in Medical Virology, 2004; 14(1) 47-64.

[41] Matsumori, A. Hepatitis C virus infection and cardiomyopathies. Circulation Research, 2005; 96(2) 144-7.

[42] Matsumori, A., et al. Myocarditis and heart failure associated with hepatitis $C$ virus infection. Journal of Cardiac Failure, 2006; 12(4) 293-8.

[43] Macejak, D.G., et al. Inhibition of hepatitis C virus (HCV)-RNA-dependent translation and replication of a chimeric $\mathrm{HCV}$ poliovirus using synthetic stabilized ribozymes. Hepatology, 2000; 31(3) 769-76.

[44] Macejak, D.G., et al. Enhanced antiviral effect in cell culture of type 1 interferon and ribozymes targeting HCV RNA. Journal of Viral Hepatitis, 2001; 8(6) 400-5. 
[45] Gonzalez-Carmona, M.A., et al. Hammerhead ribozymes with cleavage site specificity for $\mathrm{NUH}$ and $\mathrm{NCH}$ display significant anti-hepatitis $\mathrm{C}$ viral effect in vitro and in recombinant HepG2 and CCL13 cells. Journal of Hepatology, 2006; 44(6) 1017-25.

[46] Bennasser, Y., et al. Evidence that HIV-1 encodes an siRNA and a suppressor of RNA silencing. Immunity, 2005; 22(5) 607-19.

[47] Berkhout, B. and Jeang, K.T. RISCy business: MicroRNAs, pathogenesis, and viruses. Journal of Biological Chemistry, 2007; 282(37) 26641-5.

[48] Cullen, B.R. Is RNA interference involved in intrinsic antiviral immunity in mammals? Nature Immunology, 2006; 7(6) 563-7.

[49] Lecellier, C.H., et al. A cellular microRNA mediates antiviral defense in human cells. Science, 2005; 308(5721) 557-60.

[50] Otsuka, M., et al. Hypersusceptibility to vesicular stomatitis virus infection in Dicer1deficient mice is due to impaired miR24 and miR93 expression. Immunity, 2007; 27(1) 123-34.

[51] Hannon, G.J. RNA interference. Nature, 2002; 418(6894) 244-51.

[52] Tomari, Y. and Zamore, P.D. Perspective: machines for RNAi. Genes and Development, 2005; 19(5) 517-29.

[53] Caudy, A.A., et al. A micrococcal nuclease homologue in RNAi effector complexes. Nature, 2003; 425(6956) 411-4.

[54] Brennecke, J., et al. Principles of microRNA-target recognition. PLoS Biology, 2005; 3(3) e85.

[55] Grimson, A., et al. MicroRNA targeting specificity in mammals: determinants beyond seed pairing. Molecular Cell, 2007; 27(1) 91-105.

[56] Krek, A., et al. Combinatorial microRNA target predictions. Nature Genetics, 2005; 37(5) 495-500.

[57] Lewis, B.P., et al. Prediction of mammalian microRNA targets. Cell, 2003; 115(7) 787-98.

[58] Doench, J.G., Petersen, C.P., and Sharp, P.A. siRNAs can function as miRNAs. Genes and Development, 2003; 17(4) 438-42.

[59] Parker, J.S., Roe, S.M., and Barford, D. Structural insights into mRNA recognition from a PIWI domain-siRNA guide complex. Nature, 2005; 434(7033) 663-6.

[60] Yuan, J., et al. Inhibition of coxsackievirus B3 replication by small interfering RNAs requires perfect sequence match in the central region of the viral positive strand. Journal of Virology, 2005; 79(4) 2151-9. 
[61] Schubert, S., et al. Strand-specific silencing of a picornavirus by RNA interference: evidence for the superiority of plus-strand specific siRNAs. Antiviral Research, 2007; 73(3) 197-205.

[62] Zhang, H.M., et al. Targeted delivery of anti-coxsackievirus siRNAs using ligandconjugated packaging RNAs. Antiviral Research, 2009; 83(3) 307-16.

[63] Merl, S., et al. Targeting 2A protease by RNA interference attenuates coxsackieviral cytopathogenicity and promotes survival in highly susceptible mice. Circulation, 2005; 111(13) 1583-92.

[64] Racchi, G., et al. Targeting of protease 2A genome by single and multiple siRNAs as a strategy to impair CVB3 life cycle in permissive HeLa cells. Methods and Findings in Experimental and Clinical Pharmacology, 2009; 31(2) 63-70.

[65] Ahn, J., et al. A small interfering RNA targeting coxsackievirus B3 protects permissive HeLa cells from viral challenge. Journal of Virology, 2005; 79(13) 8620-4.

[66] Schubert, S., et al. Maintaining inhibition: siRNA double expression vectors against coxsackieviral RNAs. Journal of Molecular Biology, 2005; 346(2) 457-65.

[67] Werk, D., et al. Developing an effective RNA interference strategy against a plusstrand RNA virus: silencing of coxsackievirus B3 and its cognate coxsackievirus-adenovirus receptor. Biological Chemistry, 2005; 386(9) 857-63.

[68] Kim, J.Y., et al. Expression of short hairpin RNAs against the coxsackievirus B3 exerts potential antiviral effects in Cos-7 cells and in mice. Virus Research, 2007; 125(1) 9-13.

[69] Fechner, H., et al. Cardiac-targeted RNA interference mediated by an AAV9 vector improves cardiac function in coxsackievirus B3 cardiomyopathy. Journal of Molecular Medicine, 2008; 86(9) 987-97.

[70] Chau, D.H., et al. Coxsackievirus B3 proteases 2A and 3C induce apoptotic cell death through mitochondrial injury and cleavage of eIF4GI but not DAP5/p97/NAT1. Apoptosis, 2007; 12(3) 513-24.

[71] Leong, L.E., Cornell, C.T., and Semler, B.L. Processing determinants and functions of cleavage products of picornavirus polyproteins. in: Semler, B.L. and Wimmer, E. (ed.) Molecular biology of picornaviruses. Washington, D.C.: ASM Press; 2002. p187-197.

[72] Tan, E.L., Wong, A.P., and Poh, C.L. Development of potential antiviral strategy against coxsackievirus B4. Virus Research, 2010; 150(1-2) 85-92.

[73] Parsley, T.B., Cornell, C.T., and Semler, B.L. Modulation of the RNA binding and protein processing activities of poliovirus polypeptide 3CD by the viral RNA polymerase domain. Journal of Biological Chemistry, 1999; 274(18) 12867-76.

[74] Leong, L.E., Walker, P.A., and Porter, A.G. Human rhinovirus-14 protease 3C (3Cpro) binds specifically to the 5'-noncoding region of the viral RNA. Evidence that 
3Cpro has different domains for the RNA binding and proteolytic activities. Journal of Biological Chemistry, 1993; 268(34) 25735-9.

[75] Cann, A.J., editor. Principles of Molecular Virology. Waltham: Academic Press; 2005.

[76] Boden, D., et al. Human immunodeficiency virus type 1 escape from RNA interference. Journal of Virology, 2003; 77(21) 11531-5.

[77] Gitlin, L., Stone, J.K., and Andino, R. Poliovirus escape from RNA interference: short interfering RNA-target recognition and implications for therapeutic approaches. Journal of Virology, 2005; 79(2) 1027-35.

[78] Wilson, J.A. and Richardson, C.D. Hepatitis C virus replicons escape RNA interference induced by a short interfering RNA directed against the NS5b coding region. Journal of Virology, 2005; 79(11) 7050-8.

[79] Merl, S. and Wessely, R. Anti-coxsackieviral efficacy of RNA interference is highly dependent on genomic target selection and emergence of escape mutants. Oligonucleotides, 2007; 17(1) 44-53.

[80] Nygardas, M., et al. Inhibition of coxsackievirus B3 and related enteroviruses by antiviral short interfering RNA pools produced using phi6 RNA-dependent RNA polymerase. Journal of General Virology, 2009; 90(Pt 10) 2468-73.

[81] van Ooij, M.J., et al. Structural and functional characterization of the coxsackievirus B3 CRE(2C): role of CRE(2C) in negative- and positive-strand RNA synthesis. Journal of General Virology, 2006; 87(Pt 1) 103-13.

[82] Saleh, M.C., Van Rij, R.P., and Andino, R. RNA silencing in viral infections: insights from poliovirus. Virus Research, 2004; 102(1) 11-7.

[83] Lee, H.S., et al. Universal and mutation-resistant anti-enteroviral activity: potency of small interfering RNA complementary to the conserved cis-acting replication element within the enterovirus coding region. Journal of General Virology, 2007; 88(Pt 7) 2003-12.

[84] Jun, E.J., et al. Antiviral potency of a siRNA targeting a conserved region of coxsackievirus A24. Biochemical and Biophysical Research Communications, 2008; 376(2) 389-94.

[85] Kim, Y.J., et al. Recombinant lentivirus-delivered short hairpin RNAs targeted to conserved coxsackievirus sequences protect against viral myocarditis and improve survival rate in an animal model. Virus Genes, 2008; 36(1) 141-6.

[86] Lee, H.S., et al. A novel program to design siRNAs simultaneously effective to highly variable virus genomes. Biochemical and Biophysical Research Communications, 2009; 384(4) 431-5.

[87] Fechner, H., et al. Coxsackievirus B3 and adenovirus infections of cardiac cells are efficiently inhibited by vector-mediated RNA interference targeting their common receptor. Gene Therapy, 2007; 14(12) 960-71. 
[88] Crocker, S.J., et al. Amelioration of coxsackievirus B3-mediated myocarditis by inhibition of tissue inhibitors of matrix metalloproteinase-1. American Journal of Pathology, 2007; 171(6) 1762-73.

[89] Si, X., et al. Ubiquitination is required for effective replication of coxsackievirus B3. PLoS ONE, 2008; 3(7) e2585.

[90] Gao, G., et al. Proteasome activator REGgamma enhances coxsackieviral infection by facilitating p53 degradation. Journal of Virology, 2010; 84(21) 11056-66.

[91] Wong, J., et al. Autophagosome supports coxsackievirus B3 replication in host cells. Journal of Virology, 2008; 82(18) 9143-53.

[92] Bartel, D.P. MicroRNAs: target recognition and regulatory functions. Cell, 2009; 136(2) 215-33.

[93] Liu, Q. and Paroo, Z. Biochemical principles of small RNA pathways. Annual Review of Biochemistry, 2010; 79 295-319.

[94] Liu, Z., Sall, A., and Yang, D. MicroRNA: An emerging therapeutic target and intervention tool. International Journal of Molecular Sciences, 2008; 9(6) 978-99.

[95] Sall, A., et al. MicroRNAs-based therapeutic strategy for virally induced diseases. Current Drug Discovery Technologies, 2008; 5(1) 49-58.

[96] Ye, X., et al. Targeted delivery of mutant tolerant anti-coxsackievirus artificial microRNAs using folate conjugated bacteriophage Phi29 pRNA. PLoS ONE, 2011; 6(6) e21215.

[97] Wang, L., et al. MiR-342-5p suppresses coxsackievirus B3 biosynthesis by targeting the 2C-coding region. Antiviral Research, 2012; 93(2) 270-9.

[98] Furusho, K., et al. High-dose intravenous gammaglobulin for Kawasaki disease. Lancet, 1984; 2(8411) 1055-8.

[99] Latov, N., et al. Use of intravenous gamma globulins in neuroimmunologic diseases. Journal of Allergy and Clinical Immunology, 2001; 108(4 Suppl) S126-32.

[100] Weller, A.H., Hall, M., and Huber, S.A. Polyclonal immunoglobulin therapy protects against cardiac damage in experimental coxsackievirus-induced myocarditis. European Heart Journal, 1992; 13(1) 115-9.

[101] Takada, H., Kishimoto, C., and Hiraoka, Y. Therapy with immunoglobulin suppresses myocarditis in a murine coxsackievirus B3 model. Antiviral and anti-inflammatory effects. Circulation, 1995; 92(6) 1604-11.

[102] Gullestad, L., et al. Immunomodulating therapy with intravenous immunoglobulin in patients with chronic heart failure. Circulation, 2001; 103(2) 220-5.

[103] Kishimoto, C., et al. Treatment of acute inflammatory cardiomyopathy with intravenous immunoglobulin ameliorates left ventricular function associated with suppres- 
sion of inflammatory cytokines and decreased oxidative stress. International Journal of Cardiology, 2003; 91(2-3) 173-8.

[104] Fan, F., et al. Effect of PGE2 on DA tone by EP4 modulating Kv channels with different oxygen tension between preterm and term. International Journal of Cardiology, 2011; 147(1) 58-65.

[105] McNamara, D.M., et al. Controlled trial of intravenous immune globulin in recentonset dilated cardiomyopathy. Circulation, 2001; 103(18) 2254-9.

[106] Schultheiss, H.P., Kuhl, U., and Cooper, L.T. The management of myocarditis. European Heart Journal, 2011; 32(21) 2616-25.

[107] Tanaka, A., et al. An angiotensin II receptor antagonist reduces myocardial damage in an animal model of myocarditis. Circulation, 1994; 90(4) 2051-5.

[108] Sliwa, K., et al. Randomised investigation of effects of pentoxifylline on left-ventricular performance in idiopathic dilated cardiomyopathy. Lancet, 1998; 351(9109) 1091-3.

[109] Saura, M., et al. An antiviral mechanism of nitric oxide: inhibition of a viral protease. Immunity, 1999; 10(1) 21-8.

[110] Padalko, E., et al. Peroxynitrite inhibition of Coxsackievirus infection by prevention of viral RNA entry. Proceedings of the National Academy of Sciences of the United States of America, 2004; 101(32) 11731-6.

[111] Zell, R., et al. Nitric oxide donors inhibit the coxsackievirus B3 proteinases 2A and 3C in vitro, virus production in cells, and signs of myocarditis in virus-infected mice. Medical Microbiology and Immunology, 2004; 193(2-3) 91-100.

[112] Gwathmey, K., Balogun, R.A., and Burns, T. Neurologic indications for therapeutic plasma exchange: an update. Journal of Clinical Apheresis, 2011; 26(5) 261-8.

[113] Bulut, D., et al. Effects of immunoadsorption on endothelial function, circulating endothelial progenitor cells and circulating microparticles in patients with inflammatory dilated cardiomyopathy. Clinical Research in Cardiology, 2011; 100(7) 603-10.

[114] Sidwell, R.W., Robins, R.K., and Hillyard, I.W. Ribavirin: an antiviral agent. Pharmacology and Therapeutics, 1979; 6(1) 123-46.

[115] Kishimoto, C., Crumpacker, C.S., and Abelmann, W.H. Ribavirin treatment of murine coxsackievirus B3 myocarditis with analyses of lymphocyte subsets. Journal of the American College of Cardiology, 1988; 12(5) 1334-41.

[116] Harki, D.A., et al. Synthesis and antiviral activity of 5-substituted cytidine analogues: identification of a potent inhibitor of viral RNA-dependent RNA polymerases. Journal of Medicinal Chemistry, 2006; 49(21) 6166-9. 
[117] Badorff, C., et al. Nitric oxide inhibits dystrophin proteolysis by coxsackieviral protease $2 \mathrm{~A}$ through S-nitrosylation: A protective mechanism against enteroviral cardiomyopathy. Circulation, 2000; 102(18) 2276-81.

[118] Gluck, B., et al. Cardioprotective effect of NO-metoprolol in murine coxsackievirus B3-induced myocarditis. Journal of Medical Virology, 2010; 82(12) 2043-52.

[119] Ding, Y., et al. Influence of cinnamaldehyde on viral myocarditis in mice. American Journal of the Medical Sciences, 2010; 340(2) 114-20.

[120] Yun, S.H., et al. Antiviral activity of coxsackievirus B3 3C protease inhibitor in experimental murine myocarditis. Journal of Infectious Diseases, 2012; 205(3) 491-7.

[121] Fairweather, D., et al. Interferon-gamma protects against chronic viral myocarditis by reducing mast cell degranulation, fibrosis, and the profibrotic cytokines transforming growth factor-beta 1, interleukin-1 beta, and interleukin-4 in the heart. American Journal of Pathology, 2004; 165(6) 1883-94.

[122] Henke, A., et al. Direct interferon-gamma-mediated protection caused by a recombinant coxsackievirus B3. Virology, 2003; 315(2) 335-44.

[123] Daliento, L., et al. Successful treatment of enterovirus-induced myocarditis with interferon-alpha. Journal of Heart and Lung Transplantation, 2003; 22(2) 214-7.

[124] Zimmermann, O., et al. Interferon beta-1b therapy in chronic viral dilated cardiomyopathy--is there a role for specific therapy? Journal of Cardiac Failure, 2010; 16(4) 348-56.

[125] Kuhl, U., et al. Interferon-beta treatment eliminates cardiotropic viruses and improves left ventricular function in patients with myocardial persistence of viral genomes and left ventricular dysfunction. Circulation, 2003; 107(22) 2793-8.

[126] Wang, Y.X., et al. Antiviral and myocyte protective effects of murine interferon-beta and -\{alpha\}2 in coxsackievirus B3-induced myocarditis and epicarditis in Balb/c mice. American Journal of Physiology. Heart and Circulatory Physiology, 2007; 293(1) H69-76.

[127] Frustaci, A., Russo, M.A., and Chimenti, C. Randomized study on the efficacy of immunosuppressive therapy in patients with virus-negative inflammatory cardiomyopathy: the TIMIC study. European Heart Journal, 2009; 30(16) 1995-2002.

[128] Padalko, E., et al. The interferon inducer ampligen [poly(I)-poly(C12U)] markedly protects mice against coxsackie B3 virus-induced myocarditis. Antimicrobial Agents and Chemotherapy, 2004; 48(1) 267-74.

[129] Yu, Z., et al. Oral administration of interferon-alpha2b-transformed Bifidobacterium longum protects BALB/c mice against coxsackievirus B3-induced myocarditis. Virology Journal, 2011; 8525. 
[130] Ahn, J., et al. Antiviral effects of small interfering RNA simultaneously inducing RNA interference and type 1 interferon in coxsackievirus myocarditis. Antimicrobial Agents and Chemotherapy, 2012; 56(7) 3516-23.

[131] Yanagawa, B., et al. Coxsackievirus B3-associated myocardial pathology and viral load reduced by recombinant soluble human decay-accelerating factor in mice. Laboratory Investigation, 2003; 83(1) 75-85.

[132] Dorner, A., et al. Alternatively spliced soluble coxsackie-adenovirus receptors inhibit coxsackievirus infection. Journal of Biological Chemistry, 2004; 279(18) 18497-503.

[133] Lim, B.K., et al. Virus receptor trap neutralizes coxsackievirus in experimental murine viral myocarditis. Cardiovascular Research, 2006; 71(3) 517-26.

[134] Goodfellow, I.G., et al. Inhibition of coxsackie B virus infection by soluble forms of its receptors: binding affinities, altered particle formation, and competition with cellular receptors. Journal of Virology, 2005; 79(18) 12016-24.

[135] Dorner, A., et al. Treatment of coxsackievirus-B3-infected BALB/c mice with the soluble coxsackie adenovirus receptor CAR4/7 aggravates cardiac injury. Journal of Molecular Medicine, 2006; 84(10) 842-51.

[136] Demain, A.L. and Zhang, L. Natural Products and Drug Discovery. in: Zhang L, D.A. (ed.) Natural Products: Drug Discovery and Therapeutics Medicines. Totowa, NJ: Humana Press; 2005. p3-32.

[137] Zhang, L. Integrated Approaches for Discovering Novel Drugs From Microbial Natural Products. in: Zhang, L. and Demain, A.L. (ed.) Natural Products: Drug Discovery and Therapeutics Medicines. Totowa, NJ: Humana Press; 2005. p33-56.

[138] Zhang, L., et al. High-throughput synergy screening identifies microbial metabolites as combination agents for the treatment of fungal infections. Proceedings of the National Academy of Sciences of the United States of America, 2007; 104(11) 4606-11.

[139] Song, F., et al. Trichodermaketones A-D and 7-O-methylkoninginin D from the marine fungus Trichoderma koningii. Journal of Natural Products, 2010; 73(5) 806-10.

[140] Ashforth, E.J., et al. Bioprospecting for antituberculosis leads from microbial metabolites. Natural Product Reports, 2010; 27(11) 1709-19.

[141] Liu, Z.L., et al. Chinese herbal medicines for hypercholesterolemia. Cochrane Database of Systematic Reviews, 2011(7) CD008305.

[142] Zhang, Y., et al. Astragaloside IV exerts antiviral effects against coxsackievirus B3 by upregulating interferon-gamma. Journal of Cardiovascular Pharmacology, 2006; 47(2) 190-5.

[143] Chen, P., et al. Astragaloside IV attenuates myocardial fibrosis by inhibiting TGF-beta1 signaling in coxsackievirus B3-induced cardiomyopathy. European Journal of Pharmacology, 2011; 658(2-3) 168-74. 
[144] Zhang, Y., et al. Antiviral effects of sophoridine against coxsackievirus B3 and its pharmacokinetics in rats. Life Sciences, 2006; 78(17) 1998-2005.

[145] Wang, Y.F., et al. Phyllaemblicin B inhibits Coxsackie virus B3 induced apoptosis and myocarditis. Antiviral Research, 2009; 84(2) 150-8.

[146] Mohsin, S., et al. Empowering adult stem cells for myocardial regeneration. Circulation Research, 2011; 109(12) 1415-28.

[147] Soler-Botija, C., Bago, J.R., and Bayes-Genis, A. A bird's-eye view of cell therapy and tissue engineering for cardiac regeneration. Annals of the New York Academy of Sciences, 2012; 1254 57-65.

[148] Alcon, A., Cagavi Bozkulak, E., and Qyang, Y. Regenerating functional heart tissue for myocardial repair. Cellular and Molecular Life Sciences, 2012; 69(16) 2635-56.

[149] Nagaya, N., et al. Transplantation of mesenchymal stem cells improves cardiac function in a rat model of dilated cardiomyopathy. Circulation, 2005; 112(8) 1128-35.

[150] Li, J.H., Zhang, N., and Wang, J.A. Improved anti-apoptotic and anti-remodeling potency of bone marrow mesenchymal stem cells by anoxic pre-conditioning in diabetic cardiomyopathy. Journal of Endocrinological Investigation, 2008; 31(2) 103-10.

[151] Van Linthout, S., et al. Mesenchymal stem cells improve murine acute coxsackievirus B3-induced myocarditis. European Heart Journal, 2011; 32(17) 2168-78.

[152] Miteva, K., et al. Human cardiac-derived adherent proliferating cells reduce murine acute Coxsackievirus B3-induced myocarditis. PLoS ONE, 2011; 6(12) e28513.

[153] Zhang, F., et al. Cellular cardiomyoplasty for a patient with heart failure. Cardiovascular Radiation Medicine, 2003; 4(1) 43-6.

[154] Siminiak, T., et al. Autologous skeletal myoblast transplantation for the treatment of postinfarction myocardial injury: phase I clinical study with 12 months of follow-up. American Heart Journal, 2004; 148(3) 531-7.

[155] Stamm, C., et al. Autologous bone-marrow stem-cell transplantation for myocardial regeneration. Lancet, 2003; 361(9351) 45-6.

[156] Chachques, J.C., et al. Cellular cardiomyoplasty: clinical application. Annals of Thoracic Surgery, 2004; 77(3) 1121-30. 